\title{
Generalizing the Depth Relevance Condition: Deep Relevant Logics Not Included in R-Mingle
}

\author{
Gemma Robles and José M. Méndez
}

\begin{abstract}
Brady has shown how to define a class of deep relevant logics from Meyer's crystal lattice CL. The aim of this paper is to generalize Brady's result by showing how to define a class of deep relevant logics from each weak relevant matrix (weak relevant matrices only verify logics with the variable-sharing property). A class of deep relevant logics not included in R-Mingle is defined.
\end{abstract}

\section{Introduction}

As it is well known, according to Anderson and Belnap [2], the following is a necessary property of any relevant logic $\mathrm{S}$.

Definition 1.1 (Variable-sharing property-vsp, for short) $\quad$ If $A \rightarrow B$ is a theorem of $\mathrm{S}$, then $A$ and $B$ share at least one propositional variable.

In [5], Brady strengthens the vsp requiring for a formula of the form $A \rightarrow B$ to be a theorem that $A$ and $B$ share at least one propositional variable at the same depth, where "the depth of an occurrence of a subformula $B$ in a formula $A$ is roughly the number of nested $\rightarrow$ 's required to reach the occurrence of $B$ in $A$ " (see [5, p. 63]). Brady names this property the depth relevance condition (drc). And logics with the drc are named deep relevant logics. He shows that the following logic DR (and so any logic included in it) has the drc.

\section{Axioms}

$$
\begin{aligned}
& \text { (A1) } A \rightarrow A, \\
& \text { (A2) }(A \wedge B) \rightarrow A /(A \wedge B) \rightarrow B, \\
& \text { (A3) }[(A \rightarrow B) \wedge(A \rightarrow C)] \rightarrow[A \rightarrow(B \wedge C)], \\
& \text { (A4) }[(A \rightarrow B) \wedge(B \rightarrow C)] \rightarrow(A \rightarrow C), \\
& \text { (A5) } A \rightarrow(A \vee B) / B \rightarrow(A \vee B),
\end{aligned}
$$

Received October 14, 2011; accepted June 11, 2012

2010 Mathematics Subject Classification: Primary 03B47

Keywords: deep relevant logics, relevant logics, weak relevant matrices, logical matrices (C) 2014 by University of Notre Dame 10.1215/00294527-1960461 
(A6) $[(A \rightarrow C) \wedge(B \rightarrow C)] \rightarrow[(A \vee B) \rightarrow C]$,

(A7) $[A \wedge(B \vee C)] \rightarrow[(A \wedge B) \vee(A \wedge C)]$,

(A8) $\neg \neg A \rightarrow A$,

(A9) $(A \rightarrow \neg B) \rightarrow(B \rightarrow \neg A)$,

(A10) $A \vee \neg A$.

\section{Rules}

(R1) $A, A \rightarrow B \Rightarrow B$,

(R2) $A, B \Rightarrow A \wedge B$

(R3) $C \vee A, C \vee(A \rightarrow B) \Rightarrow C \vee B$,

(R4) $C \vee A \Rightarrow C \vee \neg(A \rightarrow \neg A)$,

(R5) $E \vee(A \rightarrow B), E \vee(C \rightarrow D) \Rightarrow E \vee[(B \rightarrow C) \rightarrow(A \rightarrow D)]$.

Brady's aim is to set the drc as a necessary syntactical condition for some paraconsistent logics lacking the contraction axiom, used in deriving Curry's paradox. And the logic DR "is chosen as an intuitive subsystem of DT obtained by removing the less intuitive axioms from DT" ([5, p. 64]), to wit:

(t1) $[\neg(A \rightarrow B) \rightarrow(A \rightarrow B)] \rightarrow[(B \rightarrow C) \rightarrow(A \rightarrow C)]$,

(t2) $[\neg(A \rightarrow B) \rightarrow(A \rightarrow B)] \rightarrow[(C \rightarrow A) \rightarrow(C \rightarrow B)]$,

(t3) $\neg[A \rightarrow(A \rightarrow B)] \vee(A \rightarrow B)$,

(t4) $(\neg A \rightarrow A) \rightarrow \neg(A \rightarrow \neg A)$,

(t5) $\neg A \vee(\neg A \rightarrow A)$.

Remark 1.2 DR is originally defined by introducing disjunction $\vee$ via the definition $A \vee B={ }_{\text {df }} \neg(\neg A \wedge \neg B)$.

Brady's strategy essentially consists in relativizing valuations in Meyer's crystal lattice CL to levels of depth by determining the value of outer levels in implicative formulas by valuations at inner levels. Implicative formulas are defined as follows.

Definition 1.3 (Implicative formulas) A wff $A$ is implicative iff $A$ is of the form $B \rightarrow C$, where $B$ and $C$ are wffs. (See Section 2 on the languages and logics considered in the paper.)

Meyer's CL is a simplification of Belnap's $\mathrm{M}_{0}$ used by the latter to prove for the first time that the logic of entailment $\mathrm{E}$ has the vsp (see [8, pp. 95, ff.], [3], [2, Section 22.1.3]. $\mathrm{M}_{0}$ and $\mathrm{CL}$ are displayed below in Examples 4.4 and 4.5, respectively).

The aim of this paper is to generalize Brady's strategy by using weak relevant matrices (wr-matrices). The notion of a wr-matrix is introduced in [13]. These matrices have the property that logics verified by them (see Section 2 below) have the vsp. Following Brady, it will be shown how to relativize valuations in wr-matrices in order to restrict the class of logics with the vsp verified by each particular wr-matrix to a subclass of logics with the drc.

In [13] it is proved that logics far off the spectrum of standard relevant logics have the vsp and related properties shown to be predicable of E and R by Anderson and Belnap (see [2, Section 22.1.3]). In a similar way, it is proved in the present paper that there are logics with the drc that neither include nor are included in DR but that, nevertheless, do not have the contraction axiom as a theorem. In fact, a logic with the drc not included in R-Mingle (RM) shall be defined. As it is known, R-Mingle is the result of adding the axiom "mingle" $(A \rightarrow(A \rightarrow A))$ to $\mathrm{R}$, and it lacks the vsp. Although interesting on their own, these logics with the drc not included in RM are 
mostly introduced by way of an example, because it follows from Brady's method that each wr-matrix generates a class of logics with the drc.

The structure of the paper is as follows. In Section 2, we set a series of preliminary definitions including those of logical matrix, degree of formulas, depth of a subformula within a formula, and the depth relevance condition. Section 3 is a brief discussion on the relations between the drc and the Ackermann and converse Ackermann properties. In Section 4, weak relevant matrices (wr-matrices) are defined, and it is proved that if a logic $S$ is verified by a wr-matrix, then $S$ has the vsp. In Section 5, wr-model structures are defined. Wr-model structures are built upon wrmatrices, and it is proved that any logic verified by a wr-model structure has the drc. In Section 6, a wr-matrix is displayed, verifying a class of logics not included in RM. In Section 7, a wr-model structure is built upon the wr-matrix defined in Section 6. Then, it is shown that this model structure verifies a class of deep relevant logics not included in RM3, a strong extension of RM (see [4] on RM3). Finally, in Section 8, we end the paper with some conclusions on the results obtained as well as with some comments on further work related to the present topic.

As pointed out above, our results are based on those by Brady in [5]. And we have maintained, as much as possible, Brady's notation and terminology, especially when defining wr-model structures.

\section{Logical Matrices: Preliminary Definitions}

We shall consider logics formulated in the Hilbert-style form defined on propositional languages with a set of denumerable propositional variables and some (or all) of the connectives: $\rightarrow$ (conditional), $\rightsquigarrow$ (deep relevant conditional), $\wedge$ (conjunction), $\vee$ (disjunction), $\neg$ (negation), the biconditional $\leftrightarrow$ and $\leftrightarrow \hookrightarrow$ being defined in the customary way.

The set of wff is also defined in the usual way; $A, B, C$, and so on, are metalinguistic variables.

The notion of a logical matrix is defined as follows.

Definition 2.1 A logical matrix $\mathrm{M}$ is a structure $\left(K, T, F, f_{\rightarrow}, f_{\wedge}, f_{\vee}, f_{\neg}\right)$, where

(1) $K$ is a set;

(2) $T$ and $F$ are nonempty subsets of $K$ such that $T \cup F=K$ and $T \cap F=\emptyset$;

(3) $f_{\rightarrow}, f_{\wedge}, f_{\vee}$ are binary functions (distinct from each other) on $K$, and $f_{\neg}$ is a unary function on $K$.

It is said that $K$ is the set of elements of M, $T$ is the set of designated elements, and $F$ is the set of nondesignated elements. The functions $f_{\rightarrow}, f_{\wedge}, f_{\vee}$, and $f_{\neg}$ interpret in $\mathrm{M}$ the conditional, conjunction, disjunction, and negation, respectively. In some cases, one or more of these functions may not be defined.

Now, let $\mathrm{L}$ be a propositional language, $A_{1}, \ldots, A_{n}, B$ be wff of $\mathrm{L}$, and $\mathrm{S}$ be a logic defined on L. On the other hand, let $\mathrm{M}$ be a logical matrix, and let $v_{m}$ be an assignment of elements of $\mathrm{M}$ to the propositional variables of $B$. That $B$ is assigned the element $j$ of $K$ is expressed as follows: $v_{m}(B)=j$.

Then, we set the following. 
Definition 2.2 Let $\mathrm{M}$ be a logical matrix. M verifies $B$ iff for any assignment, $v_{m}$, of elements of $K$ to the propositional variables of $B, v_{m}(B) \in T$. M falsifies $B$ iff $\mathrm{M}$ does not verify $B$.

Definition 2.3 Let $A_{1}, \ldots, A_{n} \Rightarrow B$ be a rule of derivation, and let $\mathrm{M}$ be a logical matrix. Then, $\mathrm{M}$ verifies $A_{1}, \ldots, A_{n} \Rightarrow B$ iff for any assignment, $v_{m}$, of elements of $K$ to the variables of $A_{1}, \ldots, A_{n}$ and $B$, if $v_{m}\left(A_{1}\right) \in T, \ldots, v_{m}\left(A_{n}\right) \in T$, then $v_{m}(B) \in T$. M falsifies $A_{1}, \ldots, A_{n} \Rightarrow B$ iff M does not verify $A_{1}, \ldots, A_{n} \Rightarrow B$.

Finally, we have the following definition.

Definition 2.4 Let $M$ be a logical matrix. M verifies $S$ iff $M$ verifies all axioms and rules of derivation of $\mathrm{S}$.

Remark 2.5 Formulas of the form $A \rightsquigarrow B$ are not interpreted by logical matrices but by model structures defined on wr-matrices (see Section 5 below).

Next, we shall proceed to define the depth relevance condition. In order to do this, we need (see [8, Section 11.1]) the notions of "degree of a formula $A$ " (in symbols, $\operatorname{deg}(A))$ and "depth of a formula $B$ in another formula $A$ " (in symbols, $d[B, A]$ ). Let $A$ be a wff. Then, $\operatorname{deg}(A)$ is defined inductively as follows.

\section{Definition 2.6 (Degree of formulas)}

(1) If $A$ is a propositional variable, then $\operatorname{deg}(A)=0$.

(2) If $A$ is of the form $\neg B$ and $\operatorname{deg}(B)=m$, then $\operatorname{deg}(A)=m$.

(3) If $A$ is of the form $B \vee C(B \wedge C ; B \rightarrow C), \operatorname{deg}(B)=m$, and $\operatorname{deg}(C)=n$, then $\operatorname{deg}(A)=\max \{m, n\}$.

(4) If $A$ is of the form $B \rightsquigarrow C, \operatorname{deg}(B)=m$, and $\operatorname{deg}(C)=n$, then $\operatorname{deg}(A)=\max \{m, n\}+1$.

So, the degree of a formula $A$ is the maximum number of nested $\rightsquigarrow$ 's in $A$.

Let now $A$ be a wff, and let $B$ be a subformula of $A$. Then, $d[B, A]$ is defined inductively on occurrences of $B$ in $A$ as follows.

\section{Definition 2.7 (Depth of a subformula within a formula)}

(1) We have $d[A, A]=0$.

(2) If $d[\neg B, A]=n$, then $d[B, A]=n$.

(3) If $d[B \wedge C, A](d[B \vee C, A] ; d[B \rightarrow C, A])=n$, then $d[B, A]=d[C$, $A]=n$.

(4) If $d[B \rightsquigarrow C, A]=n$, then $d[B]=d[C]=n+1$.

So, the depth of a particular occurrence of $B$ in $A$ is the number of nested $\rightsquigarrow$ 's between this particular occurrence of $B$ and the whole formula $A$. Notice that $\operatorname{deg}(A)=\{\max d[p, a] \mid p$ is a propositional variable occurring in $A\}$. That is to say, the degree of $A$ is equivalent to the depth of the propositional variable with the highest depth in $A$.

Now, the depth relevance condition is defined as follows.

Definition 2.8 (Depth relevance condition, or drc) Let $\mathrm{S}$ be a propositional logic with the following connectives: $\rightsquigarrow, \rightarrow, \wedge, \vee$, and $\neg$. S has the deep relevant condition (or $\mathrm{S}$ is deep relevant) if in all theorems of $\mathrm{S}$ of the form $A \rightsquigarrow B$ there is at least one propositional variable $p$ such that $d[p, A]=d[p, B]$. 
Example 2.9 Consider the wff $A:(p \rightsquigarrow q) \rightarrow\left[\left(\begin{array}{lll}r & \rightsquigarrow\end{array}\right) \rightarrow(t \rightsquigarrow u)\right]$. Then, $\operatorname{deg}(A)=3 ; \operatorname{deg}(p \rightsquigarrow q)=1 ; \operatorname{deg}[(r \rightsquigarrow s) \rightarrow(t \rightsquigarrow u)]=2$; $d[p \rightsquigarrow q, A]=d[(r \rightsquigarrow s) \rightarrow(t \rightsquigarrow u), A]=1 ; d[p, A]=d[q, A]=d[r \rightsquigarrow s$, $A]=d[t \rightsquigarrow u, A]=2 ; d[r, A]=d[s, A]=d[t, A]=d[u, A]=3$.

Remark 2.10 Let $\mathrm{S}$ be a propositional logic. If any of the following is a theorem of $\mathrm{S}$ and $\rightarrow$ is read as $\rightsquigarrow$, then $\mathrm{S}$ does not have the drc:

(t6) $(p \rightarrow q) \rightarrow[(q \rightarrow r) \rightarrow(p \rightarrow r)]$,

(t7) $(q \rightarrow r) \rightarrow[(p \rightarrow q) \rightarrow(p \rightarrow r)]$,

(t8) $[p \wedge(p \rightarrow q)] \rightarrow q$,

(t9) $[(p \rightarrow p) \rightarrow q] \rightarrow q$,

(t10) $p \rightarrow[(p \rightarrow q)] \rightarrow q]$,

(t11) $p \rightarrow(p \rightarrow p)$,

(t12) $[(p \rightarrow q) \rightarrow p)] \rightarrow p$,

(t13) $(p \rightarrow \neg p) \rightarrow \neg p$,

(t14) $[(p \rightarrow q) \wedge \neg q] \rightarrow \neg p$,

$(\mathrm{t} 15)[(p \rightarrow q) \wedge(p \rightarrow \neg q)] \rightarrow \neg p$.

So, notice that relevant logics such as ticket entailment, T, entailment, E, or relevance, $R$, do not have the drc.

Remark 2.11 Consider the contraction rule

(t16) $A \rightarrow(A \rightarrow B) \Rightarrow A \rightarrow B$.

Although antecedent and consequence of

$(\mathrm{t} 17)[\underline{p} \rightarrow(p \rightarrow q)] \rightarrow(\underline{p} \rightarrow q)($ when $\rightarrow$ is read as $\rightsquigarrow)$

share the underlined $p$ at the same level, (t16) cannot be a rule of any logic including $\mathrm{B}_{+}$if the drc is to be preserved because in $\mathrm{B}_{+}$plus (t16) the thesis (t8) is derivable $\left(\mathrm{B}_{+}\right.$is Routley and Meyer's basic positive logic; see [11] or [14]).

\section{Excursus: The Depth Relevance Condition and the Ackermann Property}

The Ackermann property reads as follows.

Definition 3.1 (Ackermann property) A logic S has the Ackermann property (AP) if (for any $A, B, C) A \rightarrow(B \rightarrow C)$ is unprovable in $\mathrm{S}$ if $A$ does not contain an implicative formula (see Definition 1.3).

The label "Ackermann property" is Anderson and Belnap's. The AP is named after a theorem proved by Ackermann stating that his systems $\Pi$ and $\Pi^{\prime}$ have the property (see [1, Section 6]).

On the other hand, the "converse Ackermann property" reads as follows (see [2, Section 8.12]; for results on the property, see [12] and references therein).

Definition 3.2 (Converse Ackermann property) A logic $\mathrm{S}$ has the converse Ackermann property (CAP) if (for any $A, B, C)(A \rightarrow B) \rightarrow C$ ) is unprovable in $\mathrm{S}$ if $C$ does not contain an implicative formula (see Definition 1.3).

The following is proved.

Proposition 3.3 Let $\mathrm{S}$ be a logic with the drc. Then, $\mathrm{S}$ has the AP and the CAP. 
Proof (a) $\mathrm{S}$ has the AP. Let $A \rightarrow(B \rightarrow C)$ be a wff where $A$ does not contain implicative formulas. Then, for any variable $p_{i}$ in $A, d\left[p_{i}, A\right]=0$; and for any variable $p_{i}$ in $B$ (or in $C$ ), $d\left[p_{i}, B \rightarrow C\right] \geq 1$ when $\rightarrow$ is read as $\rightsquigarrow$. So, $A$ and $B \rightarrow C$ do not share a propositional variable at the same depth. (b) $\mathrm{S}$ has the CAP. The proof is similar.

Remark 3.4 The converse of Proposition 3.3 does not hold. Consider, for example, the logic positive contractionless ticket entailment $\mathrm{TW}_{+}$. Note that $\mathrm{TW}_{+}$has the AP and the CAP (see [12]), but it does not have the drc: (t6) and (t7) in Section 2 are theorems of $\mathrm{TW}_{+}$.

\section{Weak Relevant Matrices}

First, the notion of a wr-matrix is defined.

Definition 4.1 (Weak relevant matrices or wr-matrices) $\quad$ Let $\mathrm{M}$ be a logical matrix in which $a_{F} \in F$ and $a_{1}, \ldots, a_{n}, b_{1}, \ldots, b_{m}$ are elements of $K$. And let us designate by $K_{1}$ and $K_{2}$ the subsets of $K\left\{a_{1}, \ldots, a_{n}\right\}$ and $\left\{b_{1}, \ldots, b_{m}\right\}$, respectively. The sets $K_{1}$ and $K_{2}$ are disjoint, and the members of $K_{1}$ as well as those in $K_{2}$ are possibly (but not necessarily) distinct from each other. Finally, the following conditions are fulfilled:

(1) $\forall x \forall y \in K_{1} F_{\wedge}(x, y) \& F_{\vee}(x, y) \& F_{\rightarrow}(x, y) \& F_{\neg}(x) \in K_{1}$,

(2) $\forall x \forall y \in K_{2} F_{\wedge}(x, y) \& F_{\vee}(x, y) \& F_{\rightarrow}(x, y) \& F_{\neg}(x) \in K_{2}$,

(3) $\forall x \in K_{1} \forall y \in K_{2} F_{\rightarrow}(x, y)=a_{F}$.

Then it is said that $\mathrm{M}$ is a weak relevant matrix (wr-matrix for short).

Remark 4.2 In [13], wr-matrices are introduced by a simpler definition in which $K_{1}$ and $K_{2}$ are singletons.

Then, the following is proved.

Theorem 4.3 Let $\mathrm{M}$ be a wr-matrix, and let $\mathrm{S}$ be a logic verified by $\mathrm{M}$. Then, $\mathrm{S}$ has the vsp.

Proof Assume the hypothesis of Theorem 4.3, and let $A \rightarrow B$ be a wff in which $A \rightarrow B$ do not share propositional variables. Then, let $v_{m}$ be an assignment of elements of $K$ to the variables of $A \rightarrow B$ such that $v_{m}\left(p_{n}\right)=a_{i}$ for each variable $p_{n}$ in $A$, and $v_{m}\left(p_{n}\right)=b_{l}$ for each variable $p_{n}$ in $B$, where $a_{i} \in K_{1}$ and $b_{l} \in K_{2}$. By conditions 1 and 2 in Definition 4.1, $v_{m}(A) \in K_{1}$ and $v_{m}(B) \in K_{2}$. So, $v_{m}(A \rightarrow B)=a_{F}$ by condition 3 in Definition 4.1. Therefore, if $A \rightarrow B$ is a formula in which $A$ and $B$ do not share a propositional variable, $A \rightarrow B$ is not a theorem of $\mathrm{S}$. Then Theorem 4.3 follows by contraposition. 
Example 4.4 Belnap's matrix $\mathrm{M}_{0}$ is (in another notation) the following (see [3], [2, Section 22.1.3]):

\begin{tabular}{l|llllllll|l}
$\rightarrow$ & 0 & 1 & 2 & 3 & 4 & 5 & 6 & 7 & $\neg$ \\
\hline 0 & 7 & 7 & 7 & 7 & 7 & 7 & 7 & 7 & 7 \\
1 & 0 & 6 & 0 & 6 & 0 & 0 & 6 & 7 & 6 \\
2 & 0 & 0 & 5 & 5 & 0 & 5 & 0 & 7 & 5 \\
3 & 0 & 0 & 0 & 4 & 0 & 0 & 0 & 7 & 4 \\
4 & 0 & 1 & 2 & 3 & 4 & 5 & 6 & 7 & 3 \\
5 & 0 & 0 & 2 & 2 & 0 & 5 & 0 & 7 & 2 \\
6 & 0 & 1 & 0 & 1 & 0 & 0 & 6 & 7 & 1 \\
7 & 0 & 0 & 0 & 0 & 0 & 0 & 0 & 7 & 0
\end{tabular}

\begin{tabular}{l|llllllll}
$\wedge$ & 0 & 1 & 2 & 3 & 4 & 5 & 6 & 7 \\
\hline 0 & 0 & 0 & 0 & 0 & 0 & 0 & 0 & 0 \\
1 & 0 & 1 & 0 & 1 & 0 & 0 & 1 & 1 \\
2 & 0 & 0 & 2 & 2 & 0 & 2 & 0 & 2 \\
3 & 0 & 1 & 2 & 3 & 0 & 2 & 1 & 3 \\
4 & 0 & 0 & 0 & 0 & 4 & 4 & 4 & 4 \\
5 & 0 & 0 & 2 & 2 & 4 & 5 & 4 & 5 \\
6 & 0 & 1 & 0 & 1 & 4 & 4 & 6 & 6 \\
7 & 0 & 1 & 2 & 3 & 4 & 5 & 6 & 7
\end{tabular}

\begin{tabular}{l|llllllll}
$\vee$ & 0 & 1 & 2 & 3 & 4 & 5 & 6 & 7 \\
\hline 0 & 0 & 1 & 2 & 3 & 4 & 5 & 6 & 7 \\
1 & 1 & 1 & 3 & 3 & 6 & 7 & 6 & 7 \\
2 & 2 & 3 & 2 & 3 & 5 & 5 & 7 & 7 \\
3 & 3 & 3 & 3 & 3 & 7 & 7 & 7 & 7 \\
4 & 4 & 6 & 5 & 7 & 4 & 5 & 6 & 7 \\
5 & 5 & 7 & 5 & 7 & 5 & 5 & 7 & 7 \\
6 & 6 & 6 & 7 & 7 & 6 & 7 & 6 & 7 \\
7 & 7 & 7 & 7 & 7 & 7 & 7 & 7 & 7
\end{tabular}

where
(1) $K=\{0,1,2,3,4,5,6,7\}$,
(2) $T=\{4,5,6,7\}$,
(3) $F=\{0,1,2,3\}$,
(4) $a_{1}=1$,
(5) $a_{2}=6$,
(6) $b_{1}=2$,
(7) $b_{2}=5$,
(8) $a_{F}=0$. 
Example 4.5 Meyer's crystal lattice CL is (with a little rephrasing) the following (see [8, p. 95]):

\begin{tabular}{|c|c|c|c|c|c|c|c|c|c|c|c|c|c|}
\hline$\rightarrow$ & 0 & 1 & 2 & 3 & 4 & 5 & $\neg$ & $\wedge$ & 0 & 1 & 2 & 3 & 4 \\
\hline 0 & 5 & 5 & 5 & 5 & 5 & 5 & 5 & 0 & 0 & 0 & 0 & 0 & 0 \\
\hline 1 & 0 & 4 & 0 & 0 & 0 & 5 & 4 & 1 & 0 & 1 & 2 & 3 & 4 \\
\hline 2 & 0 & 2 & 2 & 0 & 0 & 5 & 2 & 2 & 0 & 2 & 2 & 4 & 4 \\
\hline 3 & 0 & 3 & 0 & 3 & 0 & 5 & 3 & 3 & 0 & 3 & 4 & 3 & 4 \\
\hline 4 & 0 & 1 & 2 & 3 & 4 & 5 & 1 & 4 & 0 & 4 & 4 & 4 & 4 \\
\hline 5 & 0 & 0 & 0 & 0 & 0 & 5 & 0 & 5 & 0 & 1 & 2 & 3 & 4 \\
\hline
\end{tabular}

\begin{tabular}{l|llllll}
$\vee$ & 0 & 1 & 2 & 3 & 4 & 5 \\
\hline 0 & 0 & 1 & 2 & 3 & 4 & 5 \\
1 & 1 & 1 & 1 & 1 & 1 & 5 \\
2 & 2 & 1 & 2 & 1 & 2 & 5 \\
3 & 3 & 1 & 1 & 3 & 3 & 5 \\
4 & 4 & 1 & 2 & 3 & 4 & 5 \\
5 & 5 & 5 & 5 & 5 & 5 & 5
\end{tabular}

where

(1) $K=\{0,1,2,3,4,5\}$

(2) $T=\{1,2,3,4,5\}$

(3) $F=\{0\}$

(4) $a_{1}=2$,

(5) $b_{1}=3$,

(6) $a_{F}=0$.

Remark 4.6 In Examples 4.4 and $4.5, K_{1}$ and $K_{2}$ could alternatively be selected as follows: (a) $\mathrm{M}_{0} . a_{1}=2, a_{2}=5, b_{1}=1, b_{2}=6$; (b) CL. $a_{1}=3, b_{1}=2$.

\section{Wr-Model Structures and the drc}

First, wr-model structures and valuations in wr-model structures are defined.

Definition 5.1 (Wr-model structures) Let $\mathrm{M}$ be a wr-matrix. A wr-model structure $\mathrm{M}_{\mathrm{M}}$ is the set $\left\{\mathrm{M}_{0}, \mathrm{M}_{1}, \mathrm{M}_{2}, \ldots, \mathrm{M}_{n}, \ldots, \mathrm{M}_{\omega}\right\}$, where $\mathrm{M}_{0}, \mathrm{M}_{1}, \mathrm{M}_{2}, \ldots, \mathrm{M}_{n}$, $\ldots, \mathrm{M}_{\omega}$ are all identical matrices to the wr-matrix $\mathrm{M}$.

Definition 5.2 (Valuations and interpretations in a wr-model structure) A valuation $v$ in a wr-model structure $\mathrm{M}_{\mathrm{M}}$ consists of a valuation $v_{j}$ for all propositional variables, for each wr-matrix $\mathrm{M}_{j}(0 \leq j \leq \omega)$. Each $v_{j}$ assigns one of the elements of $K$ to each propositional variable. Then, each valuation $v$ is extended to an interpretation $I$ consisting of the interpretations $I_{j}$ for all formulas, for all $j$ $(0 \leq j \leq \omega)$, which are given as follows: for all propositional variables $p$ and formulas $A, B$,

(i) $I_{j}(p)=v_{j}(p)$,

(ii) $I_{j}(\neg A)=\neg\left(I_{j}(A)\right)$,

(iii) $I_{j}(A \wedge B)=I_{j}(A) \wedge I_{j}(B)$,

(iv) $I_{j}(A \vee B)=I_{j}(A) \vee I_{j}(B)$,

(v) $I_{j}(A \rightarrow B)=I_{j}(A) \rightarrow I_{j}(B)$, 
where (i)-(v) are calculated according to the wr-matrix M. In addition, formulas of the form $A \rightsquigarrow B$ are evaluated as follows $\left(a_{k} \in T^{\prime}\right.$, where $T^{\prime} \subseteq T$ in $\mathrm{M}$; see Definition 4.1):

$$
\begin{aligned}
& \text { (vi.a) } j=0 . I_{0}(A \rightsquigarrow B)=a_{k}, \\
& \text { (vi.b) } 0<j<\omega . I_{j}(A \rightsquigarrow B)=I_{j-1}(A \rightarrow B), \\
& \text { (vi.c) } j=\omega . I_{\omega}(A \rightsquigarrow B) \in T \text { iff } I_{j}(A \rightarrow B) \in T \text { for all } j(0 \leq j \leq \omega) .
\end{aligned}
$$

Then, validity is defined as follows.

Definition 5.3 (Validity in a wr-model structure) Let $\mathrm{M}_{\mathrm{M}}$ be a wr-model structure, let $B_{1}, \ldots, B_{n}, A$ be a wff, and let $\mathrm{S}$ be a logic. $A$ is valid in $\mathrm{M}_{\mathrm{M}}\left(\models_{\mathrm{M}_{\mathrm{M}}} A\right)$ iff if $I_{\omega}(A) \in T$ for all valuations $v$. The rule $B_{1}, \ldots, B_{n} \Rightarrow A$ preserves $\mathrm{M}_{\mathrm{M}}$-validity iff $I_{\omega}\left(B_{1}\right) \in T, \ldots, I_{\omega}\left(B_{n}\right) \in T$, then $I_{\omega}(A) \in T$ for all valuations $v$. Finally, $\mathrm{M}_{\mathrm{M}}$ verifies $\mathrm{S}$ iff all axioms of $\mathrm{S}$ are $\mathrm{M}_{\mathrm{M}}$-valid and all rules of $\mathrm{S}$ preserve $\mathrm{M}_{\mathrm{M}}$-validity.

Remark 5.4 Recall that $\rightarrow$ represents the conditional and that $\rightsquigarrow$ represents the deep relevant conditional (see Section 2). Actually, $\rightarrow$ is the conditional characterized by the $\rightarrow$-function in the wr-matrix, and $\rightsquigarrow$ is the conditional defined from $\rightarrow$ by clause (vi) in Definition 5.2.

Example 5.5 (see [5]) The wr-model structure $\mathrm{M}_{\mathrm{CL}}$ is the set $\left\{\mathrm{M}_{0}, \mathrm{M}_{1}, \mathrm{M}_{2}, \ldots\right.$, $\left.\mathrm{M}_{n}, \ldots, \mathrm{M}_{\omega}\right\}$, where $\mathrm{M}_{0}, \mathrm{M}_{1}, \mathrm{M}_{2}, \ldots, \mathrm{M}_{n}, \ldots, \mathrm{M}_{\omega}$ are all identical to CL (see Example 4.5), valuations are defined with respect to the set $K$ of CL, and (i)-(vi) are calculated according to the CL-functions as defined in Example $4.5\left(I_{0}(A \rightsquigarrow B)=2\right.$ for each $A, B)$. Then, axioms $\mathrm{A} 1-\mathrm{A} 10$ are $\mathrm{M}_{\mathrm{CL}}$-valid, and $\mathrm{R} 1-\mathrm{R} 5$ preserve $\mathrm{M}_{\mathrm{CL}^{-}}$ validity (see Section 1; see also [5]). Therefore, DR is a deep relevant logic: the conditional $\rightarrow$ is actually a deep relevant conditional $\rightsquigarrow$.

Now, following Brady (see [5, Theorem 1]), we show that any wr-model structure

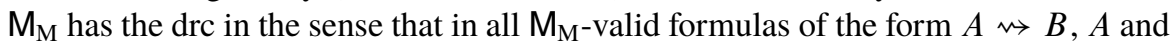
$B$ share a propositional variable at the same depth. Therefore, we will show that any wr-matrix generates a class of logics with the drc. First, we have the following.

Lemma 5.6 Let $\mathrm{M}_{\mathrm{M}}$ be a wr-model structure, and let $A \rightsquigarrow B$ be a wff such that $A$ and $B$ do not share a propositional variable at the same depth. Then, there is some $k$ for some interpretation I in $\mathrm{M}_{\mathrm{M}}$ such that for each subformula $C$ of $A, I_{k}(C) \in K_{1}$, and for each subformula $C$ of $B, I_{k}(C) \in K_{2}$.

Proof Assume the hypothesis of Lemma 5.6. Then, for all propositional variables $p$ and for all natural numbers $d, p$ does not occur at depth $d$ in $A$ or $p$ does not occur at depth $d$ in $B$. Furthermore, $\operatorname{suppose} \operatorname{deg}(A \rightsquigarrow B)=m$. Then $\operatorname{deg}(A) \leq m-1$, $\operatorname{deg}(B) \leq m-1$, and either $\operatorname{deg}(A)=m-1$ or $\operatorname{deg}(B)=m-1$. On the other hand, let $C$ be a subformula of $A$ or $B$. Then, $d[C, A]=m-1$ (or $d[C, B]=m-1$ ) is the highest depth of $C$ in $A$ (or in $B$ ). Consider now a propositional variable $p$ at depth $d$ in $A$ (or in $B$ ). Then $m-d-1$ is the measure of the distance of $d$ to the highest depth $m-1$ in $A$ (or in $B$ ). Now we set the following valuation $v$ in $\mathrm{M}_{\mathrm{M}}$ according to which all variables in $A$ and $B$ are evaluated. For each propositional variable $p$ in $A \rightsquigarrow B$ put (where $a_{i}$ and $b_{l}$ are, respectively, some fixed elements of $K_{1}$ and $K_{2}$ ):

(1) $v_{m-d-1}(p)=a_{i}$ for each depth $d$ that $p$ occurs in $A$;

(2) $v_{m-d-1}(p)=b_{l}$ for each depth $d$ that $p$ occurs in $B$;

(3) $v_{i}(p)=x_{j}$ for an arbitrary $x_{j} \in K$ if $i \geq m$, or else $i=m-d-1$ and $p$ does not occur at depth $d$ in $A$ or in $B$. 
Now, as no variable occurs at the same depth in $A$ and $B$, the valuation $v$ just defined is a consistent assignment of elements of $K$ to the variables of $A \rightsquigarrow B$. Next, following Definition 5.2 and according to the particular wr-matrix on which $\mathrm{M}_{\mathrm{M}}$ is based, $v$ is extended to an interpretation $I$. And for this interpretation $I$, the following are proved:

(4) For each subformula $C$ of $A, I_{m-d-1}(C) \in K_{1}$ for each depth $d$ that occurs in $A$.

(5) For each subformula $C$ of $B, I_{m-d-1}(C) \in K_{2}$ for each depth $d$ that $C$ occurs in $B$.

The proofs of (4) and (5) are by induction on the length of $C$. We prove (4). (The proof of (5) is similar.) Now, the cases in which $C$ is a propositional variable, a negation, a conjunction, a disjunction, or a formula of the form $A \rightarrow B$ are immediate by Definitions 4.1 and 5.2 given that $M$ is a wr-matrix (see Definition 4.1). So, let us prove the case in which $C$ is of the form $D \rightsquigarrow E$. Suppose, then, that $d[D \rightsquigarrow E, A]=d$. By Definition 2.6, $d[D, A]=d[E, A]=d+1$. By hypothesis of induction, $I_{m-(d+1)-1}(D) \in K_{1}$ and $I_{m-(d+1)-1}(E) \in K_{1}$. That is, $I_{m-d-2}(D \rightarrow E) \in K_{1}$ by condition 1 in Definition 4.1. Then, by clause (vi.b) in Definition 5.2, $I_{m-d-1}(A \rightsquigarrow B) \in K_{1}$, as was to be proved. Therefore, the interpretation $I$ defined above is the required interpretation $I$ in Lemma 5.6.

With the aid of Lemma 5.6, we shall prove that, in formulas of the form $A \rightsquigarrow B$ verified by a wr-model structure, $A$ and $B$ share a propositional variable at the same depth.

Theorem 5.7 Let $\mathrm{M}_{\mathrm{M}}$ be a wr-model structure, and suppose $\vDash_{\mathrm{M}_{\mathrm{M}}} A \rightsquigarrow B$. Then, $A$ and $B$ share at least one propositional variable at the same depth.

Proof Let $A \rightsquigarrow B$ be a wff such that $A$ and $B$ do not share a propositional variable at the same depth. By Lemma 5.6, there is some $k$ for interpretation $I$ in $\mathrm{M}_{\mathrm{M}}$ such that for every subformula $C$ of $A$ and $D$ of $B, I_{k}(C) \in K_{1}$ and $I_{k}(D) \in K_{2}$. As $A$ and $B$ are subformulas of themselves, $I_{k}(A) \in K_{1}$ and $I_{k}(B) \in K_{2}$, whence $I_{k}(A \rightarrow B)=a_{F}$ by condition (3) in Definition 4.1. So, $I_{w}(A \rightsquigarrow B) \notin T$ for this interpretation $I$ by condition (vi) in Definition 5.2. That is, $A \rightsquigarrow B$ is not valid in $\mathrm{M}_{\mathrm{M}}$. Now, Theorem 5.7 follows by contraposition.

The section is ended by exemplifying Lemma 5.6 and Theorem 5.7.

Example 5.8 Consider the wr-matrix CL defined in Example 4.5. This matrix verifies the logic $\mathrm{R}$ and, therefore, the thesis

(t10) $p \rightarrow[(p \rightarrow q) \rightarrow q]$

in Remark 2.10. Let us refer by $C, A$, and $B$ to (t10), $p$, and $(p \rightarrow q) \rightarrow q$, respectively. Then $\operatorname{deg}(C)=3, \operatorname{deg}(A)=0, \operatorname{deg}(B)=2, d[p, B]=d[\stackrel{1}{q}, B]=2$, $d[\stackrel{2}{q}, B]=1(\stackrel{1}{q}$ and $\stackrel{2}{q}$ are the first and second occurrence of $q$ in $B$, respectively). Next, it is shown that the wr-model structure $M_{C L}$ in Example 5.5 falsifies t10. We set the following valuation $v(m=3)$ :

(1) $v_{m-2-1}(p)=v_{m-2-1}(q)=3$;

(2) $v_{m-1-1}(q)=3$;

(3) $v_{m-0-1}(p)=2$. 
The assignment to $p$ in (2) and to $q$ in (3) above as well as the value of $p$ and $q$ according to (vi) of Definition 5.2 (where $m \geq 3$ ) is, for example, 1 . Then, $v$ is extended by Definition 5.2 to the corresponding interpretation $I$. According to $\mathrm{CL}$ and Definition 5.2, for this interpretation $I$, we have in succession $I_{0}(p \rightarrow q)=3, I_{1}(p \rightsquigarrow q)=3, I_{1}((p \rightsquigarrow q) \rightarrow q)=3, I_{2}((p \rightsquigarrow q) \rightsquigarrow q)=3$, $\left.\left.I_{2}(p \rightarrow[(p \rightsquigarrow q) \rightsquigarrow q)]\right)=0, I_{3}(p \rightsquigarrow[(p \rightsquigarrow q) \rightsquigarrow q)]\right)=0$. Therefore, $(\mathrm{t} 10)$ is, according to $\mathrm{M}_{\mathrm{CL}}$, not valid when $\rightarrow$ is read as $\rightsquigarrow$.

\section{A wr-Matrix Verifying Logics Not Included in R-Mingle}

Definition 6.1 Consider the matrix $\mathrm{M}_{\mathrm{DF} 6.1}=\left(K, T, F, f_{\rightarrow}, f_{\wedge}, f_{\vee}, f_{\neg}\right)$, where

(1) $K=\{0,1,2,3,4,5\}$,

(2) $T=\{1,2,3,4,5\}$,

(3) $F=\{0\}$,

(4) the functions $f_{\rightarrow}, f_{\wedge}, f_{\vee}, f_{\neg}$ are defined as shown in the tables below:

\begin{tabular}{|c|c|c|c|c|c|c|c|c|c|c|c|c|c|c|}
\hline$\rightarrow$ & 0 & 1 & 2 & 3 & 4 & 5 & $\neg$ & $\wedge$ & 0 & 1 & 2 & 3 & 4 & 5 \\
\hline 0 & 1 & 1 & 2 & 3 & 4 & 5 & 5 & 0 & 0 & 0 & 0 & 0 & 0 & 0 \\
\hline 1 & 0 & 1 & 2 & 3 & 4 & 5 & 4 & 1 & 0 & 1 & 1 & 1 & 1 & 1 \\
\hline 2 & 0 & 0 & 2 & 0 & 4 & 5 & 2 & 2 & 0 & 1 & 2 & 1 & 2 & 2 \\
\hline 3 & 0 & 0 & 0 & 3 & 4 & 5 & 3 & 3 & 0 & 1 & 1 & 3 & 3 & 3 \\
\hline 4 & 0 & 0 & 0 & 0 & 4 & 5 & 1 & 4 & 0 & 1 & 2 & 3 & 4 & 4 \\
\hline 5 & 0 & 0 & 0 & 0 & 0 & 5 & 0 & 5 & 0 & 1 & 2 & 3 & 4 & 5 \\
\hline
\end{tabular}

\begin{tabular}{l|llllll}
$\vee$ & 0 & 1 & 2 & 3 & 4 & 5 \\
\hline 0 & 0 & 1 & 2 & 3 & 4 & 5 \\
1 & 1 & 1 & 2 & 3 & 4 & 5 \\
2 & 2 & 2 & 2 & 4 & 4 & 5 \\
3 & 3 & 3 & 4 & 3 & 4 & 5 \\
4 & 4 & 4 & 4 & 4 & 4 & 5 \\
5 & 5 & 5 & 5 & 5 & 5 & 5
\end{tabular}

(5) $K_{1}=\{2\}$,

(6) $K_{2}=\{3\}$,

(7) $a_{F}=0$.

Remark 6.2 The following is a Hasse diagram of $\mathrm{M}_{\mathrm{DF6} \text {.1 }}$.

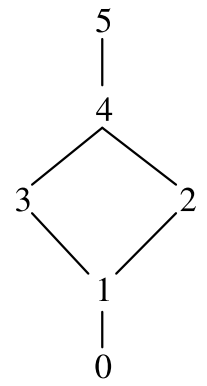

Then, we have the following. 
Proposition 6.3 $\quad \mathrm{M}_{\mathrm{DF} 6.1}$ is a wr-matrix.

Proof The proposition is proved by checking that the conditions in Definition 4.1 are fulfilled.

Proposition 6.4 Any logic verified by $\mathrm{M}_{\mathrm{DF} 6.1}$ has the vsp.

Proof This is proved by Theorem 4.3.

Now, in addition to Routley and Meyer's $B_{+}$(see [11] or [14]), $M_{D F 6.1}$ verifies, among others, the following rules and theses:

(t18) $[(A \rightarrow A) \rightarrow B] \rightarrow B$,

(t19) $[A \rightarrow(A \rightarrow B)] \leftrightarrow(A \rightarrow B)$,

(t20) $(B \rightarrow A) \rightarrow(A \rightarrow A)$,

(t21) $A \rightarrow[(B \rightarrow A) \rightarrow A]$,

(t22) $[(A \rightarrow B) \rightarrow A] \rightarrow A$,

(t23) $[(A \rightarrow B) \wedge(B \rightarrow C)] \rightarrow(A \rightarrow C)$,

(t24) $(A \rightarrow B) \rightarrow[(A \vee C) \rightarrow(B \vee C)]$,

(t25) $\neg \neg A \leftrightarrow A$,

(t26) $[(A \rightarrow B) \wedge \neg B] \rightarrow \neg A$,

(t27) $[(A \rightarrow B) \wedge(A \rightarrow \neg B)] \rightarrow \neg A$,

(t28) $\vdash A \rightarrow B \Rightarrow \vdash \neg B \rightarrow \neg A$,

(t29) $\vdash A \rightarrow B \Rightarrow \vdash(A \rightarrow \neg B) \rightarrow \neg A$.

We have the following.

Proposition 6.5 Let $\mathrm{S}$ be a logic axiomatized by adding to $B_{+}$any selection of (t18)-(t29). Then, S has the vsp.

Proof This is proved by Proposition 6.4.

Then, we note the following.

Remark 6.6 Theses (t20), (t21), (t22), and (t24) are not provable in RM3, a strong extension of R-Mingle (see [4] on RM3). Notice, by the way, that (t22) is Peirce's law, the characteristic thesis of classical implicative logic.

Therefore, $\mathrm{M}_{\mathrm{DF} 6.1}$ characterizes a class of logics with the vsp well far off the spectrum of standard relevant logics. In the following section, this matrix is used for defining deep relevant logics in which (t20) and (t24) are valid.

\section{A wr-Model Structure Built upon $\mathbf{M}_{\mathrm{DF} 6.1}$}

According to Definition 5.1, we set the following.

Definition 7.1 (The $\mathbf{M}_{\mathrm{MDF} 6.1}$-model structure) $\quad$ The wr-model structure $\mathrm{M}_{\mathrm{MDF} 6.1}$ is the set $\left\{\mathrm{M}_{0}, \mathrm{M}_{1}, \mathrm{M}_{2}, \ldots, \mathrm{M}_{n}, \ldots, \mathrm{M}_{\omega}\right\}$ where $\mathrm{M}_{0}, \mathrm{M}_{1}, \mathrm{M}_{2}, \ldots, \mathrm{M}_{n}, \ldots, \mathrm{M}_{\omega}$ are all identical to $\mathrm{M}_{\mathrm{DF6} .1}$. Then, interpretations on $\mathrm{M}_{\mathrm{MDF6.1}}$ are defined according to $\mathrm{M}_{\mathrm{DF6} .1}$ following clauses (i)-(vi) in Definition 5.2 with $a_{k}=5$. Finally, validity in $\mathrm{M}_{\mathrm{MDF} 6.1}$ is understood according to Definition 5.3.

Now, it is our aim to define deep relevant logics verified by the wr-model structure $\mathrm{M}_{\mathrm{MDF} \text {.1. }}$. But in order to do this, we follow Brady by establishing a helpful lemma. First, let us define, in addition to $T$, the following subsets of $K$ in $\mathrm{M}_{\mathrm{MDF6.1}}$ : $T *=\{5\}, a=\{2,4,5\}$, and $a *=\{3,4,5\}$. Furthermore, let us reformulate clause (vi.c) in Definition 5.2 as follows. 
Remark 7.2 Reformulation of clause (vi.c) in Definition 5.2:

(vi.c) $j=\omega$.

(1) $I_{\omega}(A \rightsquigarrow B) \in T$ iff $I_{j}(A \rightarrow B) \in T$ for all $j(0 \leq j \leq \omega)$,

(2) $I_{\omega}(A \rightsquigarrow B) \in T *$ iff $I_{j}(A \rightarrow B) \in T *$ for all $j(0 \leq j \leq \omega)$,

(3) $I_{\omega}(A \rightsquigarrow B) \in a$ iff $I_{j}(A \rightarrow B) \in a$ for all $j(0 \leq j \leq \omega)$,

(4) $I_{\omega}(A \rightsquigarrow B) \in a *$ iff $I_{j}(A \rightarrow B) \in a *$ for all $j(0 \leq j \leq \omega)$.

Then the following lemma is proved.

Lemma 7.3 For all $i(0 \leq i \leq \omega)$,

(i)
(a) $I_{i}(\neg A) \in T \Leftrightarrow I_{i}(A) \notin T *$,
(b) $I_{i}(\neg A) \in T * \Leftrightarrow I_{i}(A) \notin T$,
(c) $I_{i}(\neg A) \in a \Leftrightarrow I_{i}(A) \notin a *$,
(d) $I_{i}(\neg A) \in a * \Leftrightarrow I_{i}(A) \notin a$,

(ii)

(a) $I_{i}(A \wedge B) \in T \Leftrightarrow I_{i}(A) \in T \quad \& \quad I_{i}(B) \in T$,

(b) $I_{i}(A \wedge B) \in T * \Leftrightarrow I_{i}(A) \in T * \& I_{i}(B) \in T *$,

(c) $I_{i}(A \wedge B) \in a \Leftrightarrow I_{i}(A) \in a \quad \& \quad I_{i}(B) \in a$,

(d) $I_{i}(A \wedge B) \in a * \Leftrightarrow I_{i}(A) \in a * \& I_{i}(B) \in a *$,

(iii)

(a) $I_{i}(A \vee B) \in T \Leftrightarrow I_{i}(A) \in T$ or $I_{i}(B) \in T$,

(b) $I_{i}(A \vee B) \in T * \Leftrightarrow I_{i}(A) \in T *$ or $I_{i}(B) \in T *$,

(c) $I_{i}(A \vee B) \in a \Leftrightarrow I_{i}(A) \in$ a or $I_{i}(B) \in a$,

(d) $I_{i}(A \vee B) \in a * \Leftrightarrow I_{i}(A) \in a *$ or $I_{i}(B) \in a *$,

(iv)

(a) $I_{i}(A \rightarrow B) \in T \Leftrightarrow I_{i}(A) \in T \Rightarrow I_{i}(B) \in T$,

$$
\begin{aligned}
& \text { and } I_{i}(A) \in T * \Rightarrow I_{i}(B) \in T * \text {, } \\
& \text { and } I_{i}(A) \in a \Rightarrow I_{i}(B) \in a \text {, } \\
& \text { and } I_{i}(A) \in a * \Rightarrow I_{i}(B) \in a * \text {, } \\
& \text { (b) } I_{i}(A \rightarrow B) \in T * \Leftrightarrow I_{i}(B) \in T * \text {, } \\
& \text { (c) } I_{i}(A \rightarrow B) \in a \Leftrightarrow I_{i}(B) \in T * \text {, or } \\
& I_{i}(A) \notin T * \text { and } I_{i}(B) \in a \cap a * \text {, or } \\
& I_{i}(A) \notin a * \text { and } I_{i}(B) \in a \text { and } I_{i}(B) \notin a *, \\
& \text { (d) } I_{i}(A \rightarrow B) \in a * \Leftrightarrow I_{i}(B) \in T * \text {, or } \\
& I_{i}(A) \notin T * \text { and } I_{i}(B) \in a \cap a * \text { or } \\
& I_{i}(A) \notin a \text { and } I_{i}(B) \in a * \text { and } I_{i}(B) \notin a \text {. }
\end{aligned}
$$


Proof The lemma is proved by inspection of $\mathrm{M}_{\mathrm{DF} 6.1}$. Now, (i), (ii), and (iii) are fairly obvious. Cases (iv.b), (iv.c), and (iv.d), as well as (iv.a) from left to right are easy. So, let us show how (iv.a) from right to left can follow. Suppose then that for any wff $A, B$ and $i(0 \leq i \leq \omega)$,

$$
\begin{aligned}
& I_{i}(A) \in T \Rightarrow I_{i}(B) \in T, \\
& \quad \& I_{i}(A) \in T * \Rightarrow I_{i}(B) \in T *, \\
& \& I_{i}(A) \in a \Rightarrow I_{i}(B) \in a, \\
& \& I_{i}(A) \in a * \Rightarrow I_{i}(B) \in a * .
\end{aligned}
$$

Then, we clearly have
(a) $I_{i}(A) \notin T$ or $I_{i}(B) \in T *$, or
(b) $I_{i}(A) \notin a \& I_{i}(A) \notin a * \& I_{i}(B) \in T$, or
(c) $I_{i}(A) \notin T * \& \quad I_{i}(B) \in a \quad \& \quad I_{i}(B) \in a *$, or
(d) $I_{i}(A) \in a \quad \& \quad I_{i}(A) \notin a * \& I_{i}(B) \in a \quad \& \quad I_{i}(B) \notin a *$, or
(e) $I_{i}(A) \notin a \quad \& \quad I_{i}(A) \in a * \& I_{i}(B) \notin a \quad \& \quad I_{i}(B) \in a *$.

Now it is easy to check that if any of $a, b, c, d$, or $e$ is the case, then $I_{i}(A \rightarrow$ B) $\in T$.

Then, leaning on Lemma 7.3 , it is a simple (though tedious) task to prove the following.

Lemma 7.4 The following wff and rules of derivation are valid in the model structure $\mathrm{M}_{\mathrm{MDF} 6.1}$ :

$$
\begin{array}{ll}
(a 1) & A \rightsquigarrow A, \\
(a 2) & (A \wedge B) \rightsquigarrow A /(A \wedge B) \rightsquigarrow B, \\
(a 3) & {[(A \rightsquigarrow B) \wedge(A \rightsquigarrow C)] \rightsquigarrow[A \rightsquigarrow(B \wedge C)],} \\
(a 4) & A \rightsquigarrow(A \vee B) / B \rightsquigarrow(A \vee B), \\
(a 5) & {[(A \rightsquigarrow C) \wedge(B \rightsquigarrow C)] \rightsquigarrow[(A \vee B) \rightsquigarrow C],} \\
(a 6) & {[A \wedge(B \vee C)] \rightsquigarrow[(A \wedge B) \vee(A \wedge C)],} \\
(a 7) & (B \rightsquigarrow A) \rightsquigarrow(A \rightsquigarrow A), \\
\text { (a8) } & {[(A \rightsquigarrow B) \wedge(B \rightsquigarrow C)] \rightsquigarrow(A \rightsquigarrow C),} \\
(a 9) & (A \rightsquigarrow B) \rightsquigarrow[(A \vee C) \rightsquigarrow(B \vee C)], \\
(a 10) & A \rightsquigarrow \neg \neg A, \\
(a 11) & \neg \neg A \rightsquigarrow A, \\
(a 12) & \neg(A \wedge \neg A), \\
(\text { al3 } & A \vee \neg A, \\
(r 1) & A, A \rightsquigarrow B \Rightarrow B, \\
(r 2) & A, B \Rightarrow A \wedge B, \\
(r 3) & A \rightsquigarrow B \Rightarrow(B \rightsquigarrow C) \rightsquigarrow(A \rightsquigarrow C), \\
(r 4) & (B \rightsquigarrow C) \Rightarrow(A \rightsquigarrow B) \rightsquigarrow(A \rightsquigarrow C), \\
(r 5) & A \rightsquigarrow B \Rightarrow \neg B \rightsquigarrow \neg A,
\end{array}
$$


(r6) $A \rightsquigarrow B, A \rightsquigarrow \neg B \Rightarrow \neg A$,

(r7) $C \vee A, C \vee(A \rightsquigarrow B) \Rightarrow C \vee B$,

(r8) $C \vee A \Rightarrow C \vee \neg(A \rightsquigarrow \neg A)$,

(r9) $C \vee(A \rightsquigarrow B) \Rightarrow C \vee(\neg B \rightsquigarrow \neg A)$,

$(r 10) \quad E \vee(A \rightsquigarrow B), E \vee(C \rightsquigarrow D) \Rightarrow E \vee[(B \rightsquigarrow C) \rightarrow(A \rightsquigarrow D)]$,

(r11) $A \rightsquigarrow B$ if $A \rightarrow B$ is verified by $\mathrm{M}_{\mathrm{DF} 6.1}$ and $\rightsquigarrow$ does not appear in $A$ and $B$.

Proof We have the following:

- (a1), (a2), (a4), (a6), (a10), (a11), (a12), (a13), (r1), (r2), and (r11) are immediate;

- (a3), (a5), (a7), (a8), (a9), (r3), (r4), (r7), and (r10) are proved similarly;

- (r5), (r6), (r8), and (r9) are proved in a similar way.

So, let us prove, for example, (a9), (r5), and (r10). We use Definition 5.2 (DF5.2), Remark 7.2 (R7.2), and Lemma 7.3 (L7.3).

Now, notice that, according to Lemma 7.3, in order to show the validity in $\mathrm{M}_{\mathrm{MDF} 6.1}$ of a formula of the form $A \rightsquigarrow B$ it suffices to prove that for all valuations $v$, for all $j(0 \leq j \leq \omega)$,

(1) $I_{j}(A) \in T \Rightarrow I_{j}(B) \in T$,

(2) $I_{j}(A) \in T * \Rightarrow I_{j}(B) \in T *$,

(3) $I_{j}(A) \in a \Rightarrow I_{j}(B) \in a$,

(4) $I_{j}(A) \in a * \Rightarrow I_{j}(B) \in a *$.

(a9) is valid in $\mathrm{M}_{\mathrm{MDF} 6.1}$

Case I $I_{\omega}(A \rightsquigarrow B) \in T \Rightarrow I_{\omega}[(A \vee C) \rightsquigarrow(B \vee C)] \in T$

We prove the subcases (Ia), (Ib), and (Ic) that follow:

(Ia) $j=0 . I_{0}(A \rightsquigarrow B) \in T \Rightarrow I_{0}[(A \vee C) \rightsquigarrow(B \vee C)] \in T$.

As $I_{0}[(A \vee C) \rightsquigarrow(B \vee C)]=5$ (see Definition 7.1), $I_{0}[(A \vee C) \rightsquigarrow(B \vee C)] \in T$.

(Ib) $0<j<\omega . I_{j}(A \rightsquigarrow B) \in T \Rightarrow I_{j}[(A \vee C) \rightsquigarrow(B \vee C)] \in T$.

Suppose

$$
I_{j}(A \rightsquigarrow B) \in T \text {. }
$$

We have to prove $I_{j}[(A \vee C) \rightsquigarrow(B \vee C)] \in T$. That is,

$$
\begin{array}{lll} 
& (\mathrm{Ib} 1) & I_{j-1}(A \vee C) \in T \Rightarrow I_{j-1}(B \vee C) \in T, \\
\& & (\mathrm{Ib} 2) & I_{j-1}(A \vee C) \in T * \Rightarrow I_{j-1}(B \vee C) \in T *, \\
\& & (\mathrm{Ib} 3) \quad I_{j-1}(A \vee C) \in a \Rightarrow I_{j-1}(B \vee C) \in a, \\
\& & (\mathrm{Ib} 4) \quad I_{j-1}(A \vee C) \in a * \Rightarrow I_{j-1}(B \vee C) \in a *,
\end{array}
$$

according to DF5.2 and L7.3. By (Hyp) and DF5.2, $I_{j-1}(A \rightarrow B) \in T$. So, by L7.3,

(Ib5) $I_{j-1}(A) \in T \Rightarrow I_{j-1}(B) \in T$,

(Ib6) $I_{j-1}(A) \in T * \Rightarrow I_{j-1}(B) \in T *$,

(Ib7) $I_{j-1}(A) \in a \Rightarrow I_{j-1}(B) \in a$,

(Ib8) $I_{j-1}(A) \in a * \Rightarrow I_{j-1}(B) \in a *$.

But (Ib1)-(Ib4) are immediate from (Ib5)-(Ib8). 
(Ic) $j=\omega . I_{\omega}(A \rightsquigarrow B) \in T \Rightarrow I_{\omega}[(A \vee C) \rightsquigarrow(B \vee C)] \in T$.

Suppose $I_{\omega}(A \rightsquigarrow B) \in T$. By DF5.2, $I_{j}(A \rightarrow B) \in T$ for all $j(0 \leq j \leq \omega)$. Then, case (Ic) follows similarly as (Ib).

Case II $I_{\omega}(A \rightsquigarrow B) \in T * \Rightarrow I_{\omega}[(A \vee C) \rightsquigarrow(B \vee C)] \in T *$

Subcases (IIa) $(j=0)$ and (IIc) $(j=\omega)$ are proved similarly as in case I. So, let us prove (IIb).

(IIb) $0<j<\omega . I_{j}(A \rightsquigarrow B) \in T * \Rightarrow I_{j}[(A \vee C) \rightsquigarrow(B \vee C)] \in T *$.

Suppose $I_{j}(A \rightsquigarrow B) \in T *$. By DF5.2 and R7.2, $I_{j-1}(A \rightarrow B) \in T *$, by L7.3, $I_{j-1}(B) \in T *$, and so, $I_{j-1}(B \vee C) \in T *$. Consequently, $I_{j-1}[(A \vee C) \rightarrow$ $(B \vee C)] \in T *$ by L7.3, and $I_{j}[(A \vee C) \rightsquigarrow(B \vee C)] \in T *$ by DF5.2 and R7.2.

Case III $I_{\omega}(A \rightsquigarrow B) \in a \Rightarrow I_{\omega}[(A \vee C) \rightsquigarrow(B \vee C)] \in a$

We prove (IIIb), with subcases (IIIa) and (IIIc) being proved as above.

(IIIb) $0<j<\omega . I_{j}(A \rightsquigarrow B) \in a \Rightarrow I_{j}[(A \vee C) \rightsquigarrow(B \vee C)] \in a$.

Suppose $I_{j}(A \rightsquigarrow B) \in a$, that is, $I_{j-1}(A \rightarrow B) \in a$. By DF5.2 and R7.2,

(IIIb1) $I_{j-1}(B) \in T *$, or

(IIIb2) $I_{j-1}(A) \notin T * \& I_{j-1}(B) \in a \cap a *$, or

(IIIb3) $I_{j-1}(A) \notin a * \& I_{j-1}(B) \in a \& I_{j-1}(B) \notin a *$.

We have to prove $I_{j}[(A \vee C) \rightsquigarrow(B \vee C)] \in a$. That is,

(IIIb4) $I_{j-1}(B \vee C) \in T *$, or

(IIIb5) $I_{j-1}(A \vee C) \notin T * \& I_{j-1}(B \vee C) \in a \cap a *$, or

(IIIb6) $I_{j-1}(A \vee C) \notin a * \& I_{j-1}(B \vee C) \in a \quad \& I_{j-1}(B \vee C) \notin a *$.

We consider the three possible alternatives, (IIIb1)-(IIIb3).

1. (IIIb1). We have $I_{j-1}(B) \in T *$. Then (IIIb4) is immediate.

2. (IIIb2). We have $I_{j-1}(A) \notin T * \quad \& \quad I_{j-1}(B) \in a \cap a *$. Suppose $I_{j-1}(C) \in T *$. Then, (IIIb4) is immediate. Suppose, on the other hand, $I_{j-1}(C) \notin T *$. Then, $I_{j-1}(A \vee C) \notin T *$, whence (IIIb5) follows by $I_{j-1}(B \vee C) \in a \cap a *\left(I_{j-1}(B) \in a \cap a *\right)$.

3. (IIIb3). We have $I_{j-1}(A) \notin a * \& I_{j-1}(B) \in a \quad \& \quad I_{j-1}(B) \notin a *$. Suppose $I_{j-1}(C) \notin a *$. Then (IIIb6) is immediate. Suppose, on the other hand, $I_{j-1}(C) \in a *$. Then $I_{j-1}(B \vee C) \in a \cap a *$. Now, if $I_{j-1}(C) \in T *$, then (IIIb4) follows. And if $I_{j-1}(C) \notin T *$, then $I_{j-1}(A \vee C) \notin T *$ $\left(I_{j-1}(A) \notin a *\right)$, whence (IIIb5) follows.

Case IV $I_{w}(A \rightsquigarrow B) \in a * \Rightarrow I_{w}[(A \vee C) \rightsquigarrow(B \vee C)] \in a *$

The proof is similar to that of case (III).

With the proof of case (IV) ends the proof that (a9) is $\mathrm{M}_{\mathrm{MDF} 6.1}$-valid.

(r5) preserves $\mathrm{M}_{\mathrm{MDF} 6.1 \text {-validity }}$

Suppose

$$
I_{\omega}(A \rightsquigarrow B) \in T \text { for all } v .
$$

Let $v$ be an arbitrary valuation. We have to prove $I_{\omega}(\neg B \rightsquigarrow \neg A) \in T$. By L7.3 it suffices to prove, for this valuation, cases I, II, and III below.

Case $I j=0 . I_{0}(\neg B \rightsquigarrow \neg A) \in T$

This is immediate, as $I_{0}(\neg B \rightsquigarrow \neg A)=5$. 
Case II $0<j<\omega . I_{j}(\neg B \rightsquigarrow \neg A) \in T$

By (Hyp), $I_{j}(A \rightsquigarrow B) \in T$ for all $j$ in this $v$. So, by L7.3,

(II1) $I_{j-1}(A) \in T \Rightarrow I_{j-1}(B) \in T$,

(II2) $I_{j-1}(A) \in T * \Rightarrow I_{j-1}(B) \in T *$,

(II3) $I_{j-1}(A) \in a \Rightarrow I_{j-1}(B) \in a$,

(II4) $I_{j-1}(A) \in a * \Rightarrow I_{j-1}(B) \in a *$.

By L7.3,

(II5) $I_{j-1}(\neg B) \in T \Rightarrow I_{j-1}(\neg A) \in T$,

(II6) $I_{j-1}(\neg B) \in T * \Rightarrow I_{j-1}(\neg A) \in T *$,

(II7) $I_{j-1}(\neg B) \in a \Rightarrow I_{j-1}(\neg A) \in a$,

(II8) $I_{j-1}(\neg B) \in a * \Rightarrow I_{j-1}(\neg A) \in a *$,

whence $I_{j}(\neg B \rightsquigarrow \neg A) \in T$ follows by L7.3, DF5.2, and R7.2.

Case III $j=\omega . I_{j}(\neg B \rightsquigarrow \neg A) \in T$.

The proof of this case is similar to that of case II (see DF5.2, R7.2).

(r10) preserves $\mathrm{M}_{\mathrm{MDF} 6.1 \text {-validity }}$

Suppose that, for all $v$,

$$
I_{\omega}[E \vee(A \rightsquigarrow B)] \in T, \quad I_{w}[E \vee(C \rightsquigarrow D)] \in T .
$$

(Hyp 1)

Suppose further that, for arbitrary $v$,

$$
I_{\omega}(E) \notin T \text {. }
$$

We have to prove that $I_{j}[(B \rightsquigarrow C) \rightsquigarrow(A \rightsquigarrow D)] \in T$ for this valuation $v$. The cases where $j=0$ and $j=\omega$ are proved as in the preceding examples. So, let us prove for all $j(0<j<\omega)$ in this $v$ the following:

(I) $I_{j}(B \rightsquigarrow C) \in T \Rightarrow I_{j}(A \rightsquigarrow D) \in T$,

(II) $I_{j}(B \rightsquigarrow C) \in T * \Rightarrow I_{j}(A \rightsquigarrow D) \in T *$,

(III) $I_{j}(B \rightsquigarrow C) \in a \Rightarrow I_{j}(A \rightsquigarrow D) \in a$,

(IV) $I_{j}(B \rightsquigarrow C) \in a * \Rightarrow I_{j}(A \rightsquigarrow D) \in a *$.

As above, we use DF5.2, R7.2, and L7.3.

Now, by (Hyp 1) and (Hyp 2),

$$
\begin{gathered}
\text { (h1) } \quad I_{j-1}(A) \in T \Rightarrow I_{j-1}(B) \in T \\
\quad \& \quad I_{j-1}(C) \in T \Rightarrow I_{j-1}(D) \in T, \\
\text { (h2) } \quad I_{j-1}(A) \in T * \Rightarrow I_{j-1}(B) \in T * \\
\quad \& I_{j-1}(C) \in T * \Rightarrow I_{j-1}(D) \in T *, \\
\text { (h3) } \quad I_{j-1}(A) \in a \Rightarrow I_{j-1}(B) \in a \\
\quad \& I_{j-1}(C) \in a \Rightarrow I_{j-1}(D) \in a, \\
\text { (h4) } \quad I_{j-1}(A) \in a * \Rightarrow I_{j-1}(B) \in a * \\
\quad \& I_{j-1}(C) \in a * \Rightarrow I_{j-1}(D) \in a * .
\end{gathered}
$$

Next, we prove cases I-IV above.

Case $I I_{j}(B \rightsquigarrow C) \in T \Rightarrow I_{j}(A \rightsquigarrow D) \in T$.

Suppose $I_{j}(B \rightsquigarrow C) \in T$. Then,

(I1) $I_{j-1}(B) \in T \Rightarrow I_{j-1}(C) \in T$,

(I2) $I_{j-1}(B) \in T * \Rightarrow I_{j-1}(C) \in T *$,

(I3) $I_{j-1}(B) \in a \Rightarrow I_{j-1}(C) \in a$, 
(I4) $I_{j-1}(B) \in a * \Rightarrow I_{j-1}(C) \in a *$.

By (h1)-(h4) and (I1)-(I4), we have immediately

(I5) $I_{j-1}(A) \in T \Rightarrow I_{j-1}(D) \in T$,

(I6) $I_{j-1}(A) \in T * \Rightarrow I_{j-1}(D) \in T *$,

(I7) $I_{j-1}(A) \in a \Rightarrow I_{j-1}(D) \in a$,

(I8) $I_{j-1}(A) \in a * \Rightarrow I_{j-1}(D) \in a *$,

whence $I_{j-1}(A \rightarrow D) \in T$, and so $I_{j}(A \rightsquigarrow D) \in T$.

Case II $I_{j}(B \rightsquigarrow C) \in T * \Rightarrow I_{j}(A \rightsquigarrow D) \in T *$.

Suppose $I_{j}(B \rightsquigarrow C) \in T *$. Then, $I_{j-1}(C) \in T *$. By h2, $I_{j-1}(D) \in T *$. So, $I_{j-1}(A \rightarrow D) \in T *$, and, finally, $I_{j}(A \rightsquigarrow D) \in T *$.

Case III $I_{j}(B \rightsquigarrow C) \in a \Rightarrow I_{j}(A \rightsquigarrow D) \in a$.

Suppose $I_{j}(B \rightsquigarrow C) \in a$. Then,

(III1) $I_{j-1}(C) \in T *$, or

(III2) $I_{j-1}(B) \notin T * \& I_{j-1}(C) \in a \cap a *$, or

(III3) $I_{j-1}(B) \notin a * \& I_{j-1}(C) \in a \quad \& \quad I_{j-1}(C) \notin a *$.

We have to prove $I_{j}(A \rightsquigarrow D) \in T$. That is,

(III4) $I_{j-1}(D) \in T *$, or

(III5) $I_{j-1}(A) \notin T * \& I_{j-1}(D) \in a \cap a *$, or

(III6) $I_{j-1}(A) \notin a * \& I_{j-1}(D) \in a \quad \& \quad I_{j-1}(D) \notin a *$.

We consider each of III1, III2, and III3.

(III1) $I_{j-1}(C) \in T *$ :

Then, (III4) follows by (h2).

(III2) $I_{j-1}(B) \notin T * \& I_{j-1}(C) \in a \cap a *$ :

By (h2), $I_{j-1}(A) \notin T *$; by (h3) and (h4), $I_{j-1}(D) \in a \cap a *$. That is, (III5) is provable.

(III3) $I_{j-1}(B) \notin a * \quad \& \quad I_{j-1}(C) \in a \quad \& \quad I_{j-1}(C) \notin a *$ :

By (h4), $I_{j-1}(A) \notin a *$, and so, $I_{j-1}(A) \notin T *$. By (h3), $I_{j-1}(D) \in a$. Now, if $I_{j-1}(D) \notin a *$, we have (III6). If $I_{j-1}(D) \in a *$, (III5) follows.

Case IV $I_{j}(B \rightsquigarrow C) \in a * \Rightarrow I_{j}(A \rightsquigarrow D) \in a *$.

The proof is similar to that of case III.

With the proof that (r10) preserves $\mathrm{M}_{\mathrm{MDF6} .1}$-validity, we consider Lemma 7.4 proved.

Finally, by using Lemma 7.4, we have the following.

Theorem 7.5 Let $\mathrm{S}$ be a logic formulated with any selection of (al)-(a13) and (rl)-(r11). Then, S has the drc.

Proof This proof is immediate by Lemma 7.4.

We end this section with the following remark.

Remark 7.6 Given that (A9) of DR is not valid in $\mathrm{M}_{\mathrm{MDF6.1}}$ (it is not verified by $\mathrm{M}_{\mathrm{MDF} \text { 6.1 }}$ ), none of the logics in Theorem 7.5 includes DR. On the other hand, (a7), (a9), and (r11) are not provable in DR. So, any logic in Theorem 7.5 formulated with any of (a7), (a9), or (r11) is not included in DR. Furthermore, (a7) and (a9) are not theorems of RM3, a strong extension of R-Mingle (see [4]), as pointed out above. Therefore, logics in Theorem 7.5 formulated with (a7) and/or (a9) are deep relevant logics not included in RM and, consequently, not included in relevant logic R. 


\section{Concluding Remarks}

As was pointed out in the introduction, the drc is motivated in [5] as a necessary condition, stated in syntactic terms, for some paraconsistent logics rejecting the contraction law. But, not being a sufficient condition, the drc does not determine a unique deep relevant logic, similarly as the vsp does not determine a sole relevant logic. As we have seen, Brady's strategy is to restrict with the drc the class of logics with the vsp verified by Meyer's crystal matrix CL. And concerning this strategy, two points have to be noticed.

1. Brady chooses the logic DR (presumably an abbreviation for "depth relevance") as the preferred one among those definable from CL as indicated. Brady's choice is well motivated, as discussed below, but it has to be remarked that, given the insufficiency of the drc, the logic DR is not "maximal" in the sense that it can be extended without it losing the drc. For example, the axioms (t1), (t2), and (t4) of DT (see Section 1 above) can be added. (Axioms (t3) and (t5) are not, however, acceptable. Proofs of these facts are left to the reader.)

2. The matrix $\mathrm{CL}$ is axiomatized by adding to relevant logic $\mathrm{R}$ the following axioms (see [8, p. 95]):

$$
\begin{aligned}
& (\mathrm{CL} 1) \neg(A \wedge B) \rightarrow[(\neg A \rightarrow A) \vee(A \rightarrow B)], \\
& (\mathrm{CL} 2) A \vee(A \rightarrow B) .
\end{aligned}
$$

Now, as any logic verified by CL has the vsp and, on the other hand, (CL1) and (CL2) are acceptable in no deep relevant logic (proof is left to the reader), it is reasonable to conclude that all deep relevant logics definable from CL are included in relevant logic $\mathrm{R}$.

Brady's investigations on the topic have been pursued in [6], [7], and [9]. In [6], he provides a hierarchical (Routley-Meyer) semantics for relevant logics between Routley and Meyer's basic logic B and DR. The idea is to translate the different levels in the model structures discussed above into the Routley-Meyer semantics (see [6]). And the author concludes: "We have motivated hierarchical semantics as a semantical rendition of the Depth Relevance Condition and we are now in a position to see the close relationship that exists between these two" (see [6, p. 373]). Now, it has to be remarked that, if in [5] the interest in the drc is motivated because the property is considered a foundation for paraconsistent logics without the contraction law, in [7] the interest in the drc is justified by its own sake: as a fitter condition than the vsp to characterize relevant logics. It also has to be noted that the hierarchical Routley-Meyer semantics is adequate to some logics between B and DR but do not, of course, determine a unique deep relevant logic. On the other hand, in [7], and with much more detail in [9], a "meaning containment" semantics is defined. In this semantics, entailments are characterized by the relation of meaning containment rather than by that of meaning connection (as suggested by the vsp). This semantics is considered as a foundation of the drc "as the various depths of $\rightarrow$ would correspond to the various depths of containment sentence" (see [7, p. 172]).

In this context, it develops that the system $\mathrm{DJ}^{d}$ is the main logic. $\mathrm{DJ}^{d}$, which is, essentially, the result of deleting (A10) and (R4) from DR, is said to be the logic determined by this semantics: "Thus, the entailment of $\mathrm{DJ}^{d}$ can reasonably be said to satisfy the concept of meaning containment, expressed as a content semantics" 
(see [7, p. 171]). Consequently, it seems that it has to be concluded that $\mathrm{DJ}^{d}$ is the logic adequate to the drc. Be that as it may, it is clear that, as Brady points out, $\mathrm{DJ}^{d}$ has a number of convenient properties: it has a workable natural deduction system, and it is decidable, gentzenizable, metacomplete; it has the drc and a related hierarchical Routley-Meyer semantics and a simple consistent naive set theory (see [7, Section 8]).

As we have seen, the aim of this paper has been to generalize Brady's strategy by showing how to define a class of deep relevant logics from each weak relevant matrix. It has been shown that, given that there are weak relevant matrices verifying logics with the vsp not included in $\mathrm{R}$, there are deep relevant logics not included in $\mathrm{R}$ (actually, in R-Mingle). On the other hand, it can reasonably be expected that weak relevant matrices structurally different from those treated in this paper can be found. But it has not been our purpose to propose any of the deep relevant logics definable from $\mathrm{M}_{\mathrm{DF} 6.1}$ as an alternative to $\mathrm{DR}$ or $\mathrm{DJ}^{d}$.

We do not know if any of these logics has properties comparable to those championed by $\mathrm{DJ}^{d}$. We do not know if any of them is decidable, gentzenizable, or has a natural deduction system worthy of the name "natural". Moreover, no logic with (a7) or (a9) as an axiom is representable with a Routley-Meyer affixing-style semantics (see [15]) because these axioms belong in the category "intractable principles" discussed in [15]. Therefore, no deep relevant logic with any of the two axioms (and other similar axioms) can be given a hierarchical Routley-Meyer semantics of the type built up in [6] upon the standard affixing semantics. And, nevertheless, the logics defined in this paper and others related to them and defined upon a, in a sense, dual matrix to $\mathrm{M}_{\mathrm{DF6} .1}$ are endowed with the following properties (see [10]).

1. They can be given a neighborhood ternary semantics of the type treated in [8].

And, moreover,

2. They have a "containment semantics" of the sort defined by Brady in [7] for $\mathrm{DJ}^{d}$, this showing that the latter is not the only logic adequate to this semantics.

Both characteristics show, we think, that these logics merit consideration.

\section{References}

[1] Ackermann, W., "Begründung einer strengen Implikation," Journal of Symbolic Logic, vol. 21 (1956), pp. 113-28. MR 0080607. 111

[2] Anderson, A. R., and N. D. Belnap, Jr., Entailment, Vol. 1: The Logic of Relevance and Necessity, Princeton University Press, Princeton, 1975. MR 0406756. 107, 108, 111, 113

[3] Belnap, N. D., Jr., "Entailment and relevance," Journal of Symbolic Logic, vol. 25 (1960), pp. 144-46. MR 0141590. 108, 113

[4] Brady, R. T., "Completeness proofs for the systems RM3 and BN4," Logique et Analyse (New Series), vol. 25 (1982), pp. 9-32. Zbl 0498.03012. MR 0666984. 109, 118, 124

[5] Brady, R. T., "Depth relevance of some paraconsistent logics," Studia Logica, vol. 43 (1984), pp. 63-73. Zbl 0581.03014. MR 0782849. DOI 10.1007/BF00935740. 107, 108, 109, 115, 125

[6] Brady, R. T., "Hierarchical semantics for relevant logics," Journal of Philosophical Logic, vol. 21 (1992), pp. 357-74. MR 1187700. DOI 10.1007/BF00260741. 125, 126 
[7] Brady, R. T., "Relevant implication and the case for a weaker logic," Journal of Philosophical Logic, vol. 25 (1996), pp. 151-83. Zbl 0873.03026. MR 1391177. DOI 10.1007/BF00247002. 125, 126

[8] Brady, R. T., ed., Relevant Logics and Their Rivals, Vol. II, Ashgate Publishing, Aldershot, England, 2003. 108, 110, 114, 126

[9] Brady, R. T., Universal Logic, vol. 109 of CSLI Lecture Notes, CSLI Publications, Stanford, Calif., 2006. Zbl 1234.03010. MR 2440514. 125

[10] Méndez, J. M., G. Robles, and F. Salto, "Depth relevance, containment semantics, and the factor axioms," in preparation.

[11] Meyer, R. K., and R. Routley, "Algebraic analysis of entailment, I," Logique et Analyse (New Series), vol. 15 (1972), pp. 407-28. Zbl 0336.02020. MR 0327515. 111, 118

[12] Robles, G., "Negaciones subintuicionistas para lógicas con la conversa de la propiedad Ackermann" ("Subintuitionistic negations for logics with the converse Ackermann property"), Ph.D. dissertation, Ediciones Universidad de Salamanca, Colección Vitor 179, Salamanca, Spain, 2006. 111, 112

[13] Robles, G., and J. M. Méndez, "A general characterization of the variable-sharing property by means of logical matrices," Notre Dame Journal of Formal Logic, vol. 53 (2012), pp. 223-44. MR 2925279. 108, 112

[14] Routley, R., and R. K. Meyer, "The semantics of entailment, III," Journal of Philosophical Logic, vol. 1 (1972), pp. 192-208. Zbl 0317.02019. MR 0409116. 111, 118

[15] Routley, R., V. Plumwood, R. K. Meyer, and R. T. Brady, Relevant Logics and Their Rivals, Part I: The Basic Philosophical and Semantical Theory, Ridgeview Publishing, Atascadero, Calif., 1982. MR 0728950. 126

\section{Acknowledgments}

Authors' work supported by research project FFI2011-28494 financed by the Spanish Ministry of Economy and Competitiveness. G. Robles is supported by the Ramón y Cajal Program of the Spanish Ministry of Economy and Competitiveness. We thank the referees of the Notre Dame Journal of Formal Logic for their comments and suggestions on a previous draft of this paper.

Robles

Dpto. de Psicología, Sociología y Filosofía

Universidad de León

Campus de Vegazana, s/n

24071, León

Spain

gemmarobles@gmail.com

http://grobv.unileon.es

Méndez

Universidad de Salamanca

Campus Unamuno

Edificio FES

37007, Salamanca

Spain

sefus@usal.es

http://sites.google.com/site/sefusmendez 